\title{
Microstructuring of Optical Fibers Using a Femtosecond Laser
}

\author{
Ik-Bu Sohn*, Youngseop Kim, and Young-Chul Noh \\ Precision Optics Lab., Advanced Photonics Research Institute, GIST, Oryong-dong 1, Buk-gu, \\ Gwangju 500-712, Korea \\ Jin-Chang Ryu and Jin-Tae Kim \\ Department of Photonic Eng., Chosun University, 375 Seosuk-dong, Dong-gu, \\ Gwangju 501-759, Korea
}

(Received January 2, 2009 : revised February 5, 2009 : accepted February 6, 2009)

\begin{abstract}
Laser ablation with femtosecond lasers is highly promising for microfabrication of materials. Also, the high peak power of femtosecond lasers could induce a multiphoton absorption to ablate transparent materials. Similar results have also been were obtained in the case of optical fibers. In this paper, we present our experimental results of femtosecond laser microstructuring of optical fiber and its applications to microelectronic components and fiber optic devices. Finally, we directly produced micro holes with femtosecond laser pulses in a single step by moving an optical fiber in a preprogrammed structure. When water was introduced into a hole drilled from the bottom surface of the optical fiber, the effects of blocking and redeposition of ablated material were greatly reduced and the aspect ratio of the depth of the hole was increased. We have presented circular and rectangular-shaped holes in optical fiber.
\end{abstract}

Keywords: Femtosecond laser, Microstructuring, Optical fiber

OCIS codes : (140.3390) Laser materials processing; (060.2310) Fiber optics

\section{INTRODUCTION}

Laser micromachining is a powerful method for surface patterning and structuring of materials [1]. In recent years, microfabrication with femtosecond laser pulses has attracted many researchers' attention. When a femtosecond laser pulse is focused inside a bulk transparent material, optical breakdown induced by strongly nonlinear absorption can be localized within in the focal volume; this is a major advantage that can be used to fabricate microscale structures precisely inside samples for applications such as optical waveguides [2-4], data storage [5], photonic crystals [6], and directional couplers [7]. Drilling with femtosecond laser pulses was found to be much more controllable and reproducible than drilling with longer laser pulses [8]. Although a straight hole drilling has been investigated by many researchers, it is difficult to directly drill a three-dimensional hole because a hole is always formed in the direction parallel to the incident laser

*Corresponding author: ibson@gist.ac.kr beam. To obtain a three-dimensional hole, some researchers adopted multistep methods. Kondo and Cheng produced internal channels by moving a photomachinable glass to be perpendicular to the incident femtosecond laser beam, followed by heat treatment and subsequent etching in a dilute aqueous HF solution $[9,10]$. Li et al. fabricated a three-dimensional holes inside silica glass with femtosecond laser and water [11]. In this paper, we present a microstructure of optical fibers with femtosecond laser pulses in a single step. No post-process such as heat treatment or wet etching is needed. Finally, we show that it is possible to micromachine a micro hole in optical fibers by use of a water-assisted femtosecond laser micromachining method.

\section{EXPERIMENTS AND RESULTS}

A system setup is shown in Fig. 1. Femtosecond laser pulses of 184 -fs duration were generated from a 


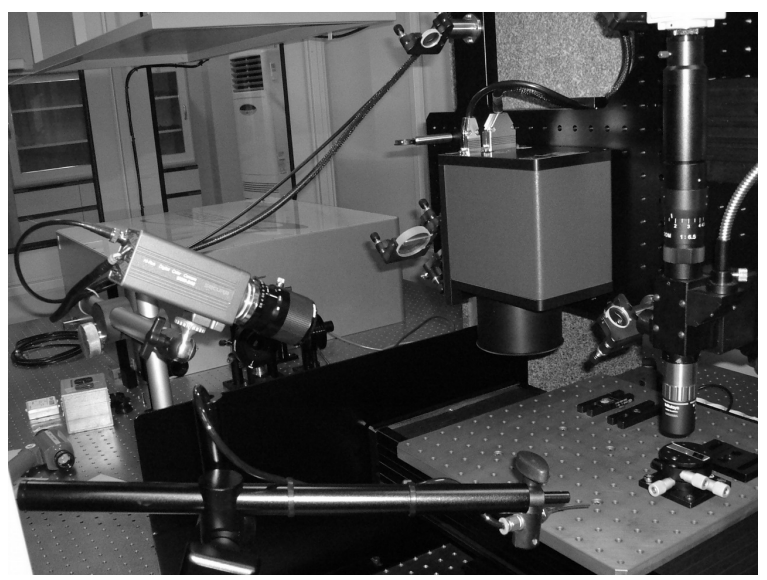

FIG. 1. Femtosecond laser pulse processing system.

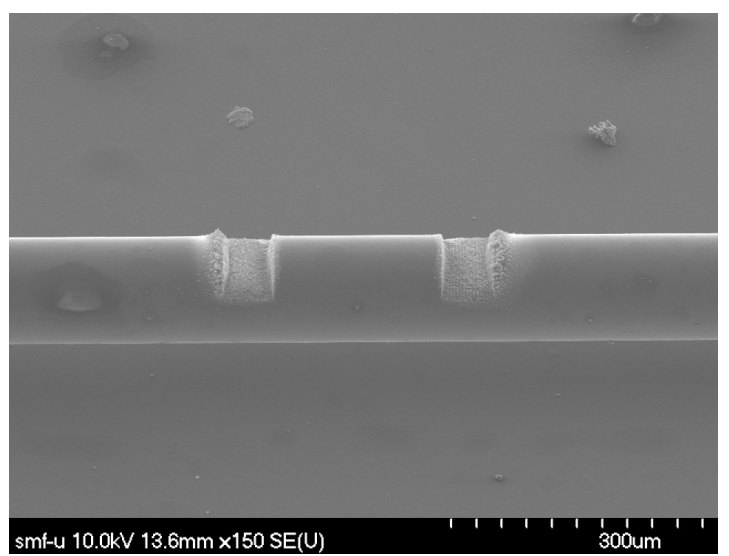

(a)

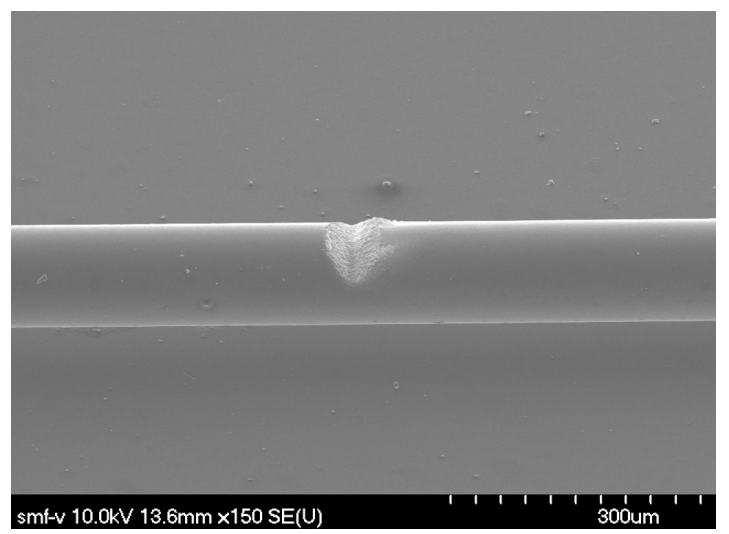

(b)

FIG. 2. SEM image of microstructured optical fiber. (a) U-grooves and (b) V-groove.

regeneratively amplified Ti:sapphire laser system at a central wavelength of $785 \mathrm{~nm}$ with $1-\mathrm{kHz}$ repetition rate. The laser beam propagated along with the optical axis and was focused into a silica glass by a microscope objective lens. Using a femtosecond laser, we fabricate periodic U-grooves on a hollow optical fiber for broadband long period gratings as shown in Fig. 2(a). One-meter- long hollow optical fiber was carved by point-by-point exposure; width of $150 \mu \mathrm{m}$, depth of $30 \mu \mathrm{m}$, and period of $550 \mu \mathrm{m}$ with 20 repeat times. The filter showed a low insertion loss of $1.7 \mathrm{~dB}$ and very broad FWHM of 160 $\mathrm{nm}$ [12]. And, we polished a photonic crystal ball lens fiber using a femtosecond laser. The side-viewing ability was provided by polishing the ball lens with a femtosecond laser to form a total internal reflection surface. The working distance and the transverse resolution of the fabricated single-body lensed-PCF were experimentally measured to be $570 \mu \mathrm{m}$ and $6.8 \mu \mathrm{m}$, respectively. With a proposed lensed-PCF probe, optical coherence tomography images of an in vitro biological sample were successfully obtained [13]. And, we fabricated V-grooved cladding on a single -mode fiber with tilted fiber Bragg gratings, which can be used as a novel low-cost fiber in-line-type bidirectional optical subassembly. V-grooved cladding can be precisely engraved on a cladding by appropriately focusing the high-power femtosecond laser beam as shown in Fig. 2(b) [14]. Also, we have successfully fabricated the microstructured surface gratings on the cleaved end of a thermally expanded core fiber by using femtosecond laser pulses. To inscribe the surface grating, the femtosecond pulsed laser beam was focused on the end surface of fiber through a 50x objective lens with NA of 0.42 and the typical pulse energy is about $0.45 \mathrm{~J}$. The one-dimensional grating structure was inscribed on the end surface of TEC fiber by translating the fiber line-by-line with a three-dimensional computer controlled stage of 100 $\mathrm{nm}$ positional accuracy in all directions. The scan speed of the focused beam was $8 \mu \mathrm{m} / \mathrm{s}$ and the grating period of the FESG could be continuously adjusted by changing the positions of the focused beam. A one-dimensional diffraction grating with a $2 \mu \mathrm{m}$ pitch was directly written on the cleaved end of TEC fiber by using focusing femtosecond laser pulses. The operation principles and fabrication procedures for inscribing fiber end surface grating structures were explained and their properties were analyzed with the measured diffraction beam patterns [15].

Finally, we demonstrated a water-assisted femtosecond laser backside etching of optical fiber. Laser-induced backside wet etching (LIBWE) is a promising technique for etching of transparent materials with high precision. The etch rate is dependent on the processing parameters and the properties of the etched surface are studied extensively [16]. In this paper, we fabricated a micro hole of optical fiber with water-assisted femtosecond laser backside processing for the first time to our best knowledge. Figure 3 shows the schematic view of the water assisted fs laser microstructuring of optical fiber and SEM image of microstructured optical fiber. Using femtosecond laser pulses with wavelength of $785.5 \mathrm{~nm}$, pulse duration of $184 \mathrm{fs}, 1 \mathrm{kHz}$ repetition rate, and N.A. value of 0.4 objective lens, we focused laser pulses on the bottom of optical fiber in water. The sample's rear 


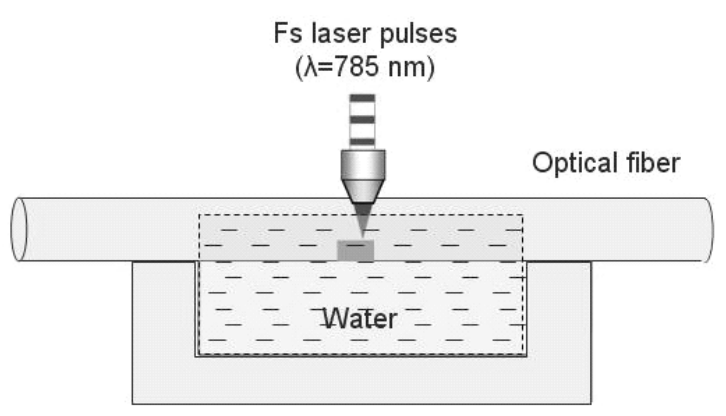

FIG. 3. Schematic view of the water assisted fs laser microstructuring of optical fiber.

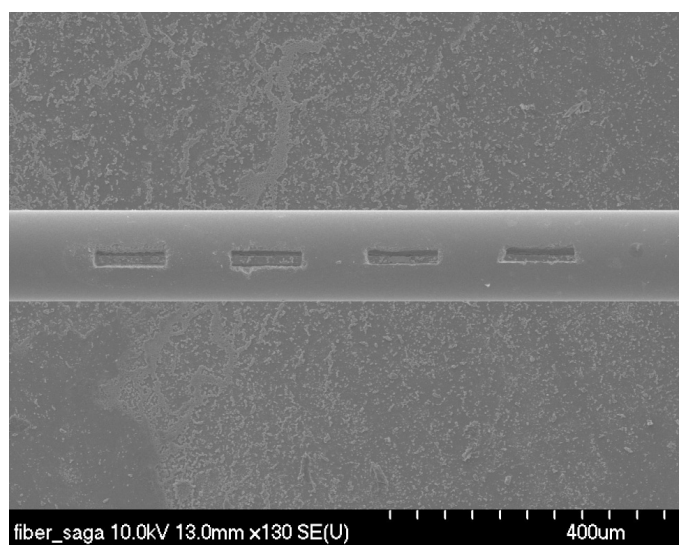

(a)

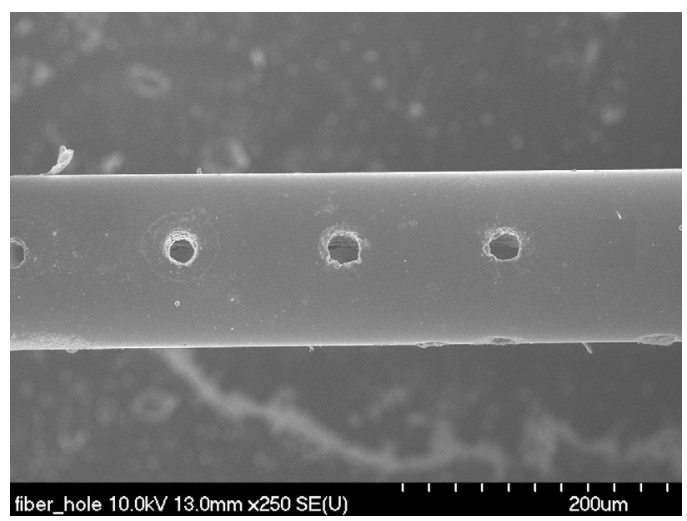

(b)

FIG. 4. SEM image of microstructured optical fiber. (a) rectangular shape, (b) circular shape.

surface was immersed in distilled water. The laser beam was first focused on the rear surface of the sample. After drilling began, we moved the focusing point toward the front surface step by step by moving the sample in the positive $\mathrm{z}$ direction. When water was introduced into a hole drilled from the bottom surface of the optical fiber, the effects of blocking and redeposition of ablated material were greatly reduced and the aspect ratio of the depth of the hole was increased. To minimize the effect of blocking and redeposition, we adopted the simple expedient of introducing water into drilled holes. When the rear surface of silica glass is in contact with water, water can flow into the hole by capillary force. Because most dust can disperse in water, the effects of redeposition and blocking are greatly reduced, and long holes can be obtained. Figure 4 shows SEM images of rectangular and circular-shaped micro holes drilled in optical fiber. We obtained the rectangular hole with $100 \mu \mathrm{m}$ $\times 20 \mu \mathrm{m}$ size micromachined by femtosecond laser with the scan speed of $100 \mu \mathrm{m} / \mathrm{s}$ and pulse energy of $10.4 \mu \mathrm{J}$ using bottom up technique in water. A single-mode fiber whose resins were stripped and a diameter is $125 \mu \mathrm{m}$ were used as transparent samples. As shown in Fig. 4, micromachining in backside water by femtosecond laser provides a clean hole while machining in air results in holes filled with debris and molten material. When we used a stronger pulse energy, the configuration of the vertical parts was damaged. Without water in the hole, we were unable to obtain tens of micrometer holes as empty as this in optical fiber. By using this method, we may drill other types of micro hole in optical fiber. This technique can be applied in the fields of microoptics, microelectronics, and microchemistry.

\section{CONCLUSION}

We experimentally presented the microstructuring of optical fibers using a femtosecond laser. V- and U-grooved optical fiber micromachined by femtosecond laser was used for long period fiber gratings and cladding mode mirror, respectively. And, we have shown that by moving a sample in a preprogrammed structure one can directly drill circular and rectangular holes of optical fiber from the rear surface of the sample with femtosecond laser pulses in a single step. When the rear surface of the sample is in contact with water, water can flow into the drilled hole to reduce the effect of blocking and redeposition of ablated material in a small hole. The aspect ratio of the depth increases greatly for the straight hole. Femtosecond laser microstructuring of optical fiber has great potential for fabrication micro optics and fiber optic devices.

\section{ACKNOWLEDGMENTS}

This work was partially supported by the Ministry of Knowledge \& Economy of through the Ultrashort Quantum Beam Facility Program and Asian Laser Center Program through a grant provided by the Gwangju Institute of Science \& Technology. 


\section{REFERENCES}

1. I.-B. Sohn, Y.-C. Noh, Y.-S. Kim, D.-K. Ko, J. Lee, and Y.-J. Choi, "Laser ablation of polypropylene films using nanosecond, picosecond, and femtosecond laser," J. Opt. Soc. Korea 12, 38-41 (2008).

2. C. B. Schaffer, A. Brodeur, J. F. Garcia, and E. Mazur, "Micromachining bulk glass by use of femtosecond laser pulses with nanojoule energy," Opt. Lett. 26, 93 (2001).

3. I.-B. Sohn, M.-S. Lee, and J.-Y. Chung, "Fabrication of optical splitter and passive alignment technique with a femtosecond laser," IEEE Photonic Technol. Lett. 17, 2349-2351 (2005).

4. Y.-R. Lee, N. E. Yu, C. Jung, B.-A. Yu, D.-K. Ko, I. -B. Sohn, and J.-M. Lee, "Second-harmonic generation in periodically poled lithium niobate waveguides fabricated by femtosecond laser pulses," Appl. Phys. Lett. 89, 171103 (2006).

5. E. N. Glezer, M. Milosavljevic, L. Huang, R. J. Finlay, T.-H. Her, J. P. Callan, and E. Mazur, "Three-dimensional optical storage inside transparent materials," Opt. Lett. 21, 2023 (1996).

6. H.-B. Sun, Y. Xu, S. Juodkazis, K. Sun, M. Watanabe, S. Matsuo, H. Misawa, and J. Nishii, "Arbitrary-lattice photonic crystals created by multiphoton microfabrication," Opt. Lett. 26, 325 (2001).

7. C. Florea and K. A. Winick, "Fabrication and characterization of photonic devices directly written in glass using femtosecond laser pulses," J. Lightwave Technol. 21, 246-253 (2003).

8. B. N. Chichkov, C. Momma, S. Nolte, F. Von Alvensleben, and A. Tunnermann, "Femtosecond, picosecond and nano- second laser ablation of solids," Appl. Phys. A 63, 109 (1996).

9. Y. Kondo, J. Qiu, T. Mitsuyu, K. Hirao, and T. Yoko, "Three-dimensional microdrilling of glass by multiphoton process and chemical etching,” Jpn. J. Appl. Phys. 38, L1146 (1999).

10. Y. Cheng, K. Sugioka, and K. Midorikawa, "Microfluidic laser embedded in glass by three-dimensional femtosecond laser microprocessing," Opt. Lett. 29, 2007 (2004).

11. Y. Li, K. Itoh, W. Watanabe, K. Yamada, D. Kuroda, J. Nishii, and Y. Jiang, "Three-dimensional hole drilling of silica glass from the rear surface with femtosecond laser pulses," Opt. Lett. 26, 1912 (2001).

12. W. Ha, Y. Jung, J. Kim, W. Shin, I.-B. Sohn, D. Ko, J. Lee, and K. Oh, "Broadband long period grating on hollow optical fiber with femtosecond laser pulses," in Proc. OECC (Yokohama, Japan, July 2007), paper 13C3-2.

13. H. Y. Choi, S. Y. Ryu, J. Na, B. H. Lee, I.-B. Sohn, Y.-C. Noh, and J. Lee, "Single-body lensed photonic crystal fibers as side-viewing probes for optical imaging systems," Opt. Lett. 33, 34 (2008).

14. K.-S. Lim, J. J. Lee, S. Lee, S. Yoon, C. H. Yu, I.-B. Sohn, and H. S. Kang, "A novel low-cost fiber in-linetype bidirectional optical subassembly," IEEE Photon. Technol. Lett. 19, 1233 (2007).

15. W. Shin, I.-B. Sohn, B. A. Yu, Y. L. Lee, S. C. Choi, Y. C. Noh, J. Lee, and D. K. Ko, "Microstructured fiber end surface grating for coarse WDM signal monitoring," IEEE Photon. Technol. Lett. 19, 550 (2007).

16. K. Zimmer, R. Bohme, D. Ruthe, and B. Rauschenbach, "Backside laser etching of fused silica using liquid gallium," Appl. Phys. A 84, 455 (2006). 